\title{
Article \\ Detection of Human Cholangiocarcinoma Markers in Serum Using Infrared Spectroscopy
}

\author{
Patutong Chatchawal ${ }^{1,2,3}$, Molin Wongwattanakul ${ }^{2,3,4}$, Patcharaporn Tippayawat ${ }^{2,4}$, Kamilla Kochan ${ }^{5}($, \\ Nichada Jearanaikoon ${ }^{6}$, Bayden R. Wood ${ }^{5, *}$ and Patcharee Jearanaikoon ${ }^{2,3,4, *}$ (i) \\ 1 Biomedical Sciences, Graduate School, Khon Kaen University, Khon Kaen 40002, Thailand; \\ patutongc@kkumail.com \\ 2 Center for Research and Development of Medical Diagnosis Laboratories, \\ Faculty of Associated Medical Sciences, Khon Kaen University, Khon Kaen 40002, Thailand; \\ moliwo@kku.ac.th (M.W.); patchatip@kku.ac.th (P.T.) \\ 3 Cholangiocarcinoma Research Institute, Khon Kaen University, Khon Kaen 40002, Thailand \\ 4 Center for Innovation and Standard for Medical Technology and Physical Therapy, \\ Faculty of Associated Medical Sciences, Khon Kaen University, Khon Kaen 40002, Thailand \\ 5 Center for Biospectroscopy, School of Chemistry, Faculty of Science, Monash University, Monash, \\ VIC 3800, Australia; Kamila.Kochan@monash.edu \\ 6 Synchrotron Light Research Institute, Nakhon Ratchasima 30000, Thailand; nichada@slri.or.th \\ * Correspondence: Bayden.wood@monash.edu (B.R.W.); patjea@kku.ac.th (P.J.)
}

check for

updates

Citation: Chatchawal, P.;

Wongwattanakul, M.; Tippayawat, P.; Kochan, K.; Jearanaikoon, N.; Wood, B.R.; Jearanaikoon, P. Detection of Human Cholangiocarcinoma Markers in Serum Using Infrared Spectroscopy. Cancers 2021, 13, 5109. https:/ / doi.org/10.3390/cancers13205109

Academic Editor: Fiona M. Lyng

Received: 16 September 2021

Accepted: 8 October 2021

Published: 12 October 2021

Publisher's Note: MDPI stays neutral with regard to jurisdictional claims in published maps and institutional affiliations.

Copyright: (c) 2021 by the authors. Licensee MDPI, Basel, Switzerland This article is an open access article distributed under the terms and conditions of the Creative Commons Attribution (CC BY) license (https:// creativecommons.org/licenses/by/ $4.0 /)$.
Simple Summary: Cholangiocarcinoma is a form of liver cancer that is found, predominantly, in Thailand. Due to the non-specific symptoms and laboratory investigation, it is difficult to rule out cholangiocarcinoma from other liver conditions. Here, we demonstrate the development of a diagnostic tool for cholangiocarcinoma, based on the ATR-FTIR analyses of sera, coupled with multivariate analyses and machine learning tools to obtain a better specificity. The innovative approach that shows highly promising results for this otherwise difficult to diagnose cancer.

Abstract: Cholangiocarcinoma (CCA) is a malignancy of the bile duct epithelium. Opisthorchis viverrini infection is a known high-risk factor for CCA and in found, predominantly, in Northeast Thailand. The silent disease development and ineffective diagnosis have led to late-stage detection and reduction in the survival rate. Attenuated total reflectance-Fourier transform infrared spectroscopy (ATR-FTIR) is currently being explored as a diagnostic tool in medicine. In this study, we apply ATR-FTIR to discriminate CCA sera from hepatocellular carcinoma (HCC), biliary disease (BD) and healthy donors using a multivariate analysis. Spectral markers differing from healthy ones are observed in the collagen band at 1284,1339 and $1035 \mathrm{~cm}^{-1}$, the phosphate band (vsPO $\mathrm{O}_{2}^{-}$) at $1073 \mathrm{~cm}^{-1}$, the polysaccharides band at $1152 \mathrm{~cm}^{-1}$ and $1747 \mathrm{~cm}^{-1}$ of lipid ester carbonyl. A Principal Component Analysis (PCA) shows discrimination between CCA and healthy sera using the $1400-1000 \mathrm{~cm}^{-1}$ region and the combined $1800-1700+1400-1000 \mathrm{~cm}^{-1}$ region. Partial Least Square-Discriminant Analysis (PLS-DA) scores plots in four of five regions investigated, namely, the $1400-1000 \mathrm{~cm}^{-1}, 1800-1000 \mathrm{~cm}^{-1}, 3000-2800+1800-1000 \mathrm{~cm}^{-1}$ and $1800-1700+1400-1000 \mathrm{~cm}^{-1}$ regions, show discrimination between sera from CCA and healthy volunteers. It was not possible to separate CCA from HCC and BD by PCA and PLS-DA. CCA spectral modelling is established using the PLS-DA, Support Vector Machine (SVM), Random Forest (RF) and Neural Network (NN). The best model is the NN, which achieved a sensitivity of $80-100 \%$ and a specificity between 83 and $100 \%$ for CCA, depending on the spectral window used to model the spectra. This study demonstrates the potential of ATR-FTIR spectroscopy and spectral modelling as an additional tool to discriminate CCA from other conditions.

Keywords: cholangiocarcinoma (CCA); attenuated total reflectance-Fourier transform infrared (ATRFTIR) spectroscopy; hepatocellular carcinoma (HCC); biliary disease (BD); multivariate analysis; machine learning 


\section{Introduction}

Cholangiocarcinoma (CCA) is a malignancy arising from the bile duct epithelium, which is found, sporadically, all over the world. CCA incidence in western countries was reported between 0.3 and 3.36 per 100,000 people, while in eastern countries, the rate is even higher. The highest incidence was found in Northeast Thailand, which reported $85-118.5$ cases per 100,000 people with a high prevalence in Khon Kaen [1,2]. The disease can be caused by various risk factors-primary sclerosing cholangitis, cholelithiasis, biliary disorders, hepatitis $B$ and $C$ infection and lifestyle-related risk, e.g., alcohol consumption and cigarette smoking-, while liver fluke infection (Opisthorchis viverrini and Clonorchis sinensis) is reported as a common risk of CCA in east Asia [3,4]. Approximately, $10 \%$ of chronically infected patients will develop CCA after 30-40 years [2,4].

CCA patients generally have no symptoms, while a long-standing infection and inflammation cause non-specific symptoms, including malaise, jaundice, cholangitis, hepatomegaly, upper quadrant abdominal pain, fatigue, etc. [5]. Unfortunately, a physical examination cannot distinguish CCA from these particular symptoms due to the similarity to other hepatobiliary diseases, especially hepatocellular carcinoma (HCC). Imaging techniques (ultrasound, magnetic resonance imaging (MRI), magnetic resonance cholangiopancreatography (MRCP), computerized tomography (CT) scan) are used to investigate CCA by detecting biliary obstruction, biliary stricture and mass forming. However, these techniques are limited by the cancer itself, as the accuracy depends on the type of tumor, anatomical lesion and tumor size [6]. Laboratory investigations performed by measuring liver function and tumor markers in patient serum are nonspecific for CCA because liver enzymes and bilirubin levels can be elevated in hepatic disorders, while CA19-9 levels can also be found in GI tract cancers [7]. A pathological examination of stained biopsy tissue is the most precise technique and is currently used as a confirmation method. Nevertheless, this technique requires an invasive sample collection, complicated sample handling, time consumingsample preparation and is labor intensive, which is not suitable for CCA screening or large-scale studies. Potential tumor markers for CCA screening and diagnosis are still intensively investigated in the research process; however, most of these markers require a complicated sample processing and analysis [8]. Although a combination of markers may provide more accurate results [9], the analysis of all markers of interest renders a high cost and is time consuming.

Attenuated Total Reflectance-Fourier Transform Infrared (ATR-FTIR) spectroscopy can be used to detect molecular vibrations of molecules in complex biological samples, including serum [10], which contain a lot of biomolecular information that is useful for a health status assessment. ATR-FTIR spectroscopy has been used to detect cancer-specific biomarkers in serum [11]. Advantages of the ATR-FTIR technique include the ease of sample manipulation and a short measurement time $(2 \mathrm{~min})$. Furthermore, ATR-FTIR is a reagent-less technique, requiring only small volumes of a sample that produce a highsignal-to noise ratio output for a further chemometric analysis. Additionally, a single scan of the sample can provide spectral information associated with the molecular phenotype of the disease agent and/or host response [12].

Vibrational spectroscopy, coupled with machine learning algorithms, has previously been applied to sera samples for various diseases, providing an excellent discrimination against controls [13,14]. A study comparing ATR spectra of sera from breast cancer patients versus heathy sera using a Neural Network reported $92-95 \%$ sensitivity and $95-100 \%$ specificity with the main spectral changes observed in the $\mathrm{CH}$ stretching band, $\mathrm{C}-\mathrm{O}$ from the ribose backbone and P-O vibrations [15]. Toraman et al. [16] applied ATR-FTIR spectroscopy to investigate plasma from colon cancer patients using the multilayer perceptron Neural Network and Support Vector Machine. They reported 76-93\% sensitivity, 97-100\% specificity using the Neural Network and a $63-90 \%$ sensitivity, $80-95 \%$ specificity with the SVM [16]. An ATR-FTIR study on sera from patients with brain cancer using SVM reported $93.3 \%$ sensitivity and $92.8 \%$ specificity [17]. These studies set a precedence for diagnosing other cancers from sera samples with ATR-FTIR spectroscopy. 
In our previous study, we reported FTIR spectral discrimination between cholangiocarcinoma and normal tissues and serum samples using an animal model [18]. The discrimination was based on changes in the phosphodiester bands, amino acid, carboxylic ester and collagen molecules in tissue and serum, whereas additional bands corresponding to the amide I, II, polysaccharides and nucleic acid molecules were important in discriminating serum samples from CCA and controls [18]. In this study, we apply ATR-FTIR spectroscopy to investigate human clinical serum samples with the aim to develop a model to discriminate the spectra of CCA from healthy, hepatocellular carcinoma (HCC) and biliary disease (BD) sera using chemometrics. Partial Least Squares Discriminant Analysis (PLS-DA), Support Vector Machine (SVM), Random Forest (RF) and Neural Network (NN) models are established and evaluated by calculating \% accuracy, \% sensitivity and $\%$ specificity.

\section{Materials and Methods}

\subsection{Human Sera}

Sixty samples of CCA, twenty samples of HCC and twenty samples of BD sera were supplied by the Cholangiocarcinoma Research Institute (CARI), Faculty of Medicine, Khon Kaen University, Khon Kaen, Thailand. Fifty healthy sera samples were left over from health checkup program at the Community Medical Laboratory, Faculty of Associated Medical Sciences, Khon Kaen University. Human samples were approved for use by the Center for Ethics in Human Research, Khon Kaen University (HE601117). All sera were aliquoted and kept at $-20^{\circ} \mathrm{C}$ prior to analyses.

\subsection{ATR-FTIR Spectroscopy for Serum Analysis}

Eight microliters of healthy, CCA, HCC and BD sera was deposited on aluminum foil, air dried and measured using a portable Agilent ATR-FTIR spectrometer 4500 series (Agilent technologies, CA, USA). The parameters for sera measurement were 64 co-added scans for both background and sample, $4 \mathrm{~cm}^{-1}$ spectral resolution in the $4000-650 \mathrm{~cm}^{-1}$ spectral range with 4 replicates for each sample.

\subsection{ATR-FTIR Spectral Preprocessing and Analysis}

ATR-FTIR spectra acquired from healthy, CCA, HCC and BD human sera were preprocessed by calculating the 2nd derivatives with 15 smoothing points using SavitzkyGolay algorithm and unit vector normalization. Multivariate analysis was performed in 5 spectral ranges: (1) $3000-2800 \mathrm{~cm}^{-1}$, (2) $1800-1000 \mathrm{~cm}^{-1}$, (3) $1400-1000 \mathrm{~cm}^{-1}$ and combine regions, including (4) $1800-1700+1400-1000 \mathrm{~cm}^{-1}$ and (5) $3000-2800+1800-1000 \mathrm{~cm}^{-1}$. PCA was performed using The Unscrambler ${ }^{\circledR} \mathrm{X}$ (version 10.5, Camo Software, Oslo, Norway). Two-thirds of the samples acquired from each group were categorized as a calibration set to perform supervised analysis, including PLS-DA (The Unscrambler ${ }^{\circledR}$ $\mathrm{X}$ version 10.5, Camo Software), Support Vector machine (SVM) (Quasar version 0.9.0, University of Ljubljana, Slovenia), Random Forest (RF) and Neural Network (NN) using multilayer perceptron (Weka software version 3.8.4, The University of Waikato, Hamilton, New Zealand), while averaged spectra from another $1 / 3$ of the samples were appended as a validation set to predict the established model and calculate $\%$ accuracy, $\%$ sensitivity and $\%$ specificity. No technical replicates from the same sample were included in both the training and test set to avoid over optimistic modeling, i.e., the technical replicate trap.

\subsection{Method Evaluation and Calculation}

Predictive results of each model were assigned in Table 1 for comparison of the clinical diagnoses and index test results. Percent accuracy, sensitivity and specificity were calculated by following Formula:

$$
\% \text { Accuracy }=\left(\frac{a+d}{a+b+c+d}\right) \times 100
$$




$$
\begin{aligned}
& \% \text { Sensitivity }=\left(\frac{a}{a+c}\right) \times 100 \\
& \% \text { Specificity }=\left(\frac{d}{b+d}\right) \times 100
\end{aligned}
$$

Table 1. Table defines the prediction performance between reference and index tests.

\begin{tabular}{ccc}
\hline $\begin{array}{c}\text { Index Test } \\
\text { (Predictive Model) }\end{array}$ & CCA & $\begin{array}{c}\text { Clinical Diagnoses } \\
\text { Other Condition }\end{array}$ \\
\hline CCA & $a$ & $b$ \\
Other condition & $c$ & $d$ \\
\hline
\end{tabular}

\section{Results}

\subsection{Characteristic Peaks of Healthy, CCA, HCC and BD Spectra}

Averaged 2nd derivative spectra of healthy, CCA, HCC and BD sera from the CH stretching region (3000-2800 $\left.\mathrm{cm}^{-1}\right)$ and fingerprint spectral region $\left(1800-1000 \mathrm{~cm}^{-1}\right)$ are shown in Figure 1a,b, respectively. A spectral shift from $1289 \mathrm{~cm}^{-1}$ in the healthy group to $1284 \mathrm{~cm}^{-1}$ in CCA, HCC and BD was observed, which indicated an alteration of collagen molecules. A shoulder at $\sim 106$ and $\sim 1046 \mathrm{~cm}^{-1}$ was found in the healthy group assigned to the $\mathrm{C}-\mathrm{O}$ stretching and bending vibration in the $\mathrm{C}-\mathrm{OH}$ group of carbohydrate (Figure 1c) [19].
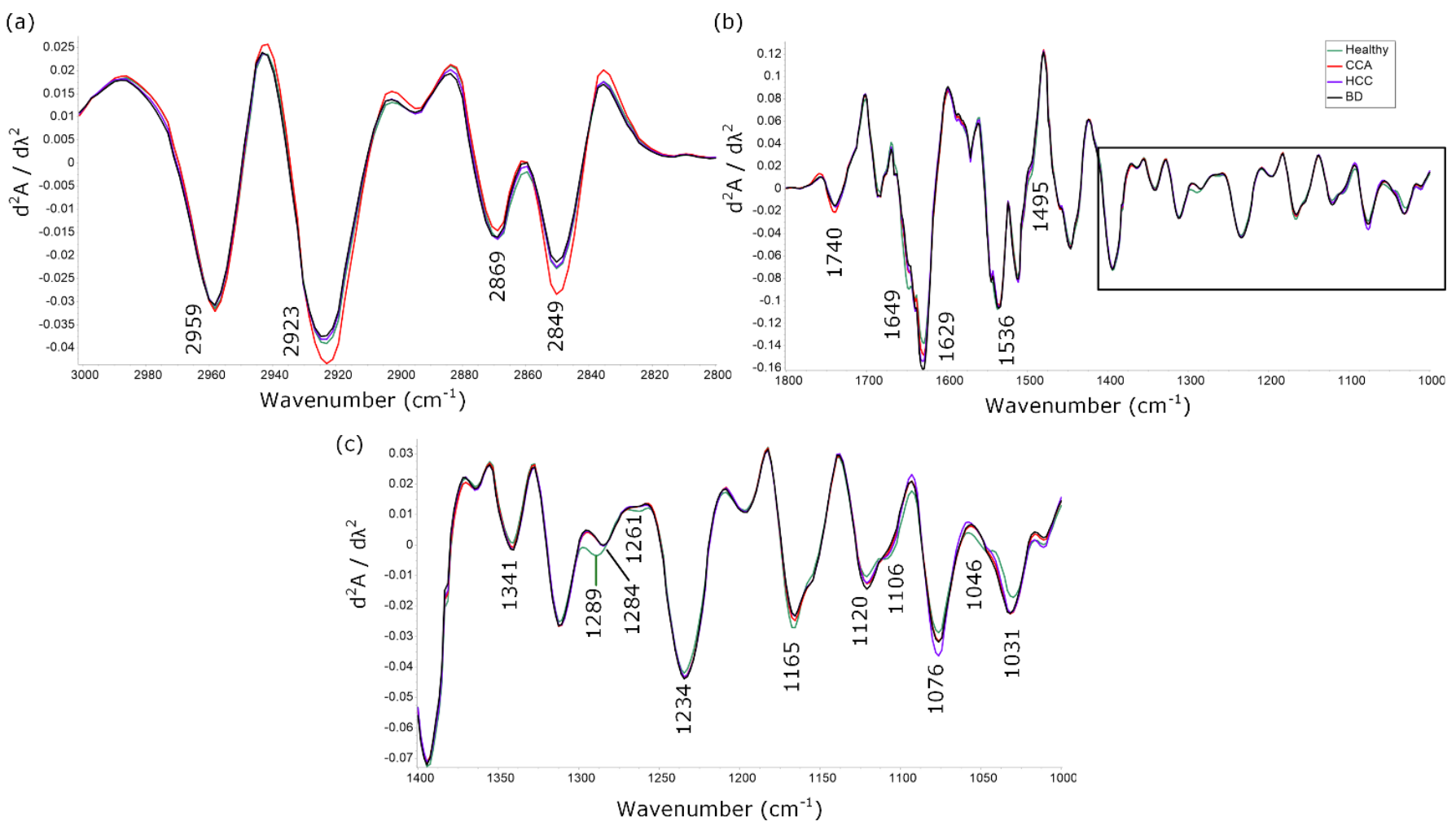

Figure 1. 2nd derivative of averaged spectra from healthy (green), CCA (red), HCC (purple) and BD (black) sera in (a) CH stretching region (3000-2800 $\left.\mathrm{cm}^{-1}\right)$, (b) fingerprint spectral $\left(1800-1000 \mathrm{~cm}^{-1}\right)$ and (c) $1400-1000 \mathrm{~cm}^{-1}$ region.

However, a clear spectral difference could not be observed in the $\mathrm{CH}$ stretching region and amide I, II region. The averaged spectra in certain regions may not provide informative data due to their common functional molecule or the abundant protein found in serum; hence, chemometric approaches were required to further interrogate the data. 


\subsection{CCA Spectral Discrimination Using Unsupervised Analysis: Principal Component Analysis (PCA)}

Spectra acquired from $60 \mathrm{CCA}$ and 50 healthy serum samples were preprocessed and analyzed using the Principal Component Analysis (PCA). The PCA scores plot for the $1400-1000 \mathrm{~cm}^{-1}$ region showed discrimination along PC1 (Figure 2a), while the combined regions $1800-1700+1400-1000 \mathrm{~cm}^{-1}$ showed discrimination along PC2 (Figure 2c). The loadings plot for the $1400-1000 \mathrm{~cm}^{-1}$ region (Figure $2 \mathrm{~b}$ ) showed wavenumber values at $1372,1338,1309,1227,1152,1116,1072$ and $1035 \mathrm{~cm}^{-1}$ corresponding to the CCA sera. The additional wavenumber value at $1747 \mathrm{~cm}^{-1}$ of CCA sera was observed when using the combined region $\left(1747,1370,1339,1310,1227,1152,1116,1073\right.$ and $1035 \mathrm{~cm}^{-1}$ ) (Figure 2d). Peaks at $\sim 1072$ and $~ 1116 \mathrm{~cm}^{-1}$ were assigned to P-O-C modes from phosphodiester groups in nucleic acids. The discrimination was also based on variance in the amide III region that included contributions from collagen $\left(\sim 1400-1200 \mathrm{~cm}^{-1}\right.$ and $\left.1035 \mathrm{~cm}^{-1}\right)$ derived from $\mathrm{CH}_{2}$ bending and in-plane of $\mathrm{NH}$ bending and $\mathrm{CN}$ stretching vibrations of the protein and $\mathrm{CH}_{2}$ of collagen [20,21]. Bands at $1152 \mathrm{~cm}^{-1}$ along with $\sim 1073 \mathrm{~cm}^{-1}$ can be indicated as glycogen or polysaccharide molecules [22]. The ester carbonyl band in CCA appeared at $1747 \mathrm{~cm}^{-1}$, while, in uninfected patients, it shifted to $1733 \mathrm{~cm}^{-1}$. A band at $1702 \mathrm{~cm}^{-1}$ was assigned to the $\mathrm{C}=\mathrm{O}$ vibration of nucleic bases [23].
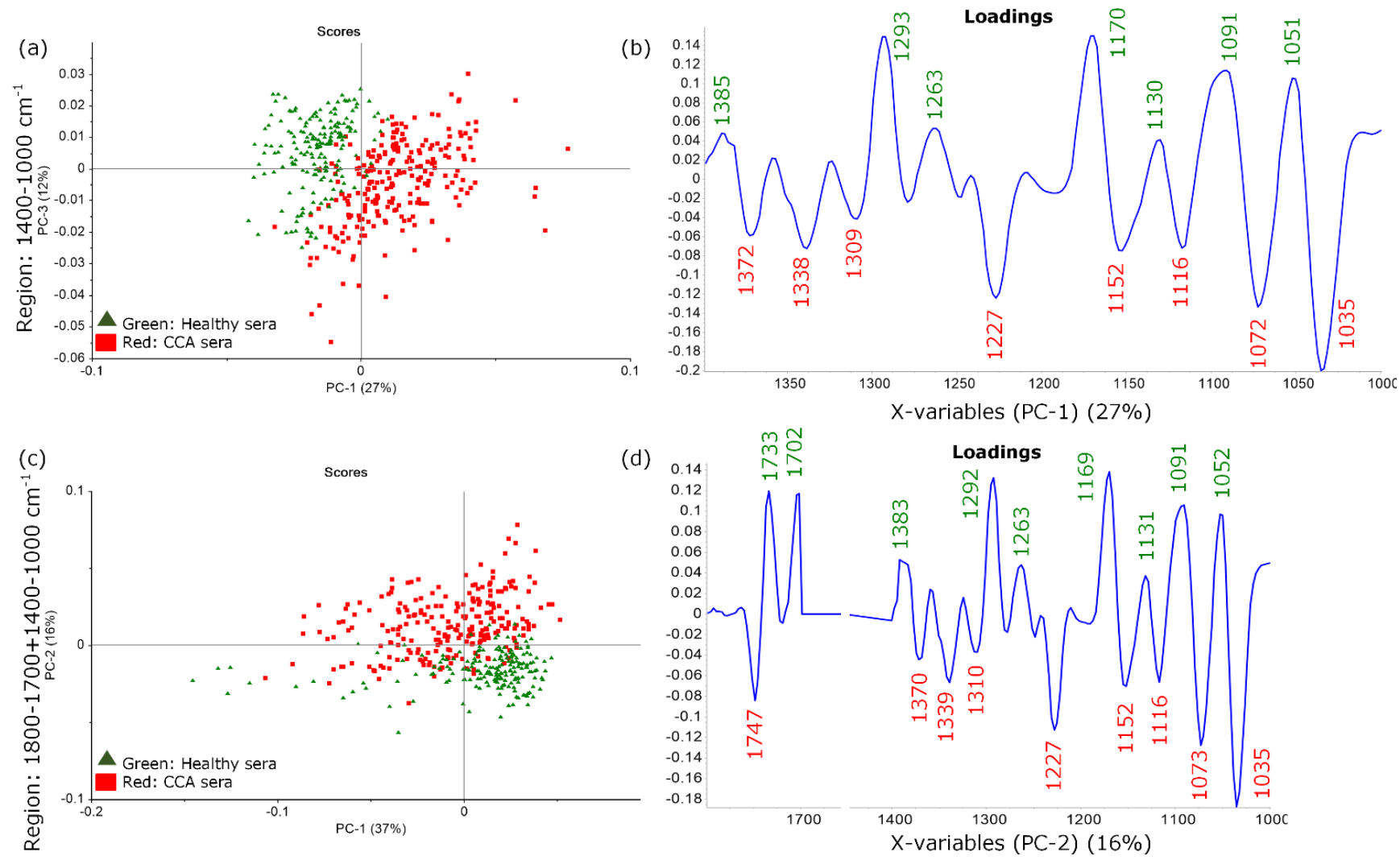

Figure 2. PCA results of healthy (green) and CCA (red) sera displayed in (a) PC-1,3 scores plot in the $1400-1000 \mathrm{~cm}^{-1} \mathrm{spectral}^{-1}$ region, (b) corresponding PC-1 loadings plot and (c) PC-1,2 scores plot using the combined 1800-1700 $+1400-1000 \mathrm{~cm}^{-1}$ spectral regions and (d) corresponding PC-2 loadings plot.

A PCA analysis was also performed in the $\mathrm{CH}$ stretching region $\left(3000-2800 \mathrm{~cm}^{-1}\right)$, fingerprint spectral region (1800-1000 $\mathrm{cm}^{-1}$ ) and a combination of the $3000-2800+1800-1000 \mathrm{~cm}^{-1}$ spectral regions. However, the PCA scores plots showed no discrimination among the two groups of sera (Figure S1). Furthermore, the spectra of $20 \mathrm{HCC}, 20 \mathrm{BD}$ and $60 \mathrm{CCA}$ sera were evaluated with a PCA analysis. The PCA scores plots showed no discrimination among HCC vs. CCA and BD vs. CCA sera (Figure S2). A supervised analysis using the 
Partial Least Squares Discriminant Analysis (PLS-DA) was then applied to provide a better discrimination and establish the PLS-DA calibration model for CCA sera.

\subsection{Establishment and Evaluation of CCA Predictive Model Using Partial Least Squares Discriminant Analysis (PLS-DA)}

Spectra were categorized into two groups: a calibration set and a prediction set. Forty of the CCA and thirty-five of the healthy sera were modeled in the calibration set and analyzed using PLS-DA to generate a PLS predictive model. The averaged spectra of the remaining samples (20 CCA and 15 healthy) were predicted using the generated PLS model for various spectral regions. The sensitivity and specificity for each of the spectral regions are shown in Table 2. The PLS model in the fingerprint spectral region (1800-1000 $\mathrm{cm}^{-1}$ ) showed discrimination along PC1 ( $x$-axis) (Figure S3a). The regression coefficients (Figure S3b) showed wavenumber values from the $1743 \mathrm{~cm}^{-1} \mathrm{C}=\mathrm{O}$ lipid ester carbonyl, 1687, 1665, 1630 and $1555 \mathrm{~cm}^{-1}$ from $\mathrm{C}=\mathrm{O}$ and $\mathrm{N}-\mathrm{H}$ vibrational modes of proteins, $1512 \mathrm{~cm}^{-1}$ of N-H or C-N vibrations and the combination of polysaccharide, glycogen, amide III, collagen and phosphodiester modes from nucleic acids at lower wavenumber values $\left(1450,1408,1371,1337,1307,1277,1246,1225,1154,1117,1074\right.$ and $\left.1034 \mathrm{~cm}^{-1}\right)$ corresponding to CCA sera samples. The PLS model in $1400-1000 \mathrm{~cm}^{-1}$ spectral region showed a clear discrimination along Factor-1 ( $x$-axis) (Figure $3 a)$. The regression coefficients plot (Figure $3 \mathrm{~b}$ ) appeared to have a similar profile to PLS-DA performed on the $1800-1000 \mathrm{~cm}^{-1}$ region. Moreover, the discrimination trend could also be found in the combined region of $1800-1700+1400-1000 \mathrm{~cm}^{-1}$ (Figure S3c) and 3000-2800 + 1800-1000 $\mathrm{cm}^{-1}$ (Figure S3e), while the $\mathrm{CH}$ stretching region alone $\left(3000-2800 \mathrm{~cm}^{-1}\right)$ showed no discrimination between the two groups (Figure S3d).
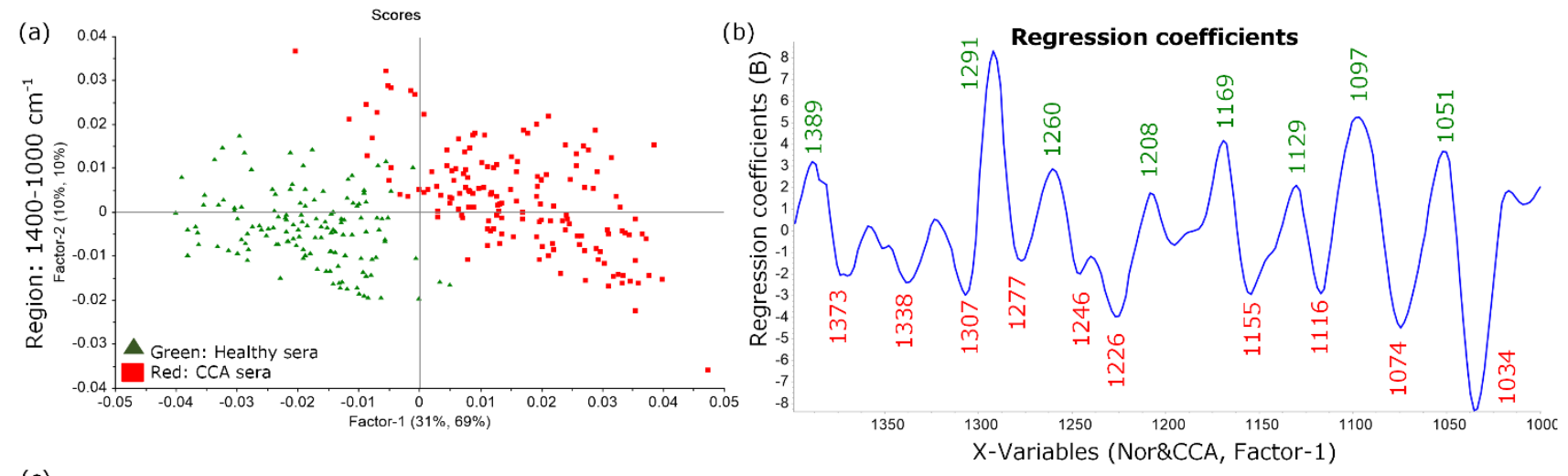

(c)

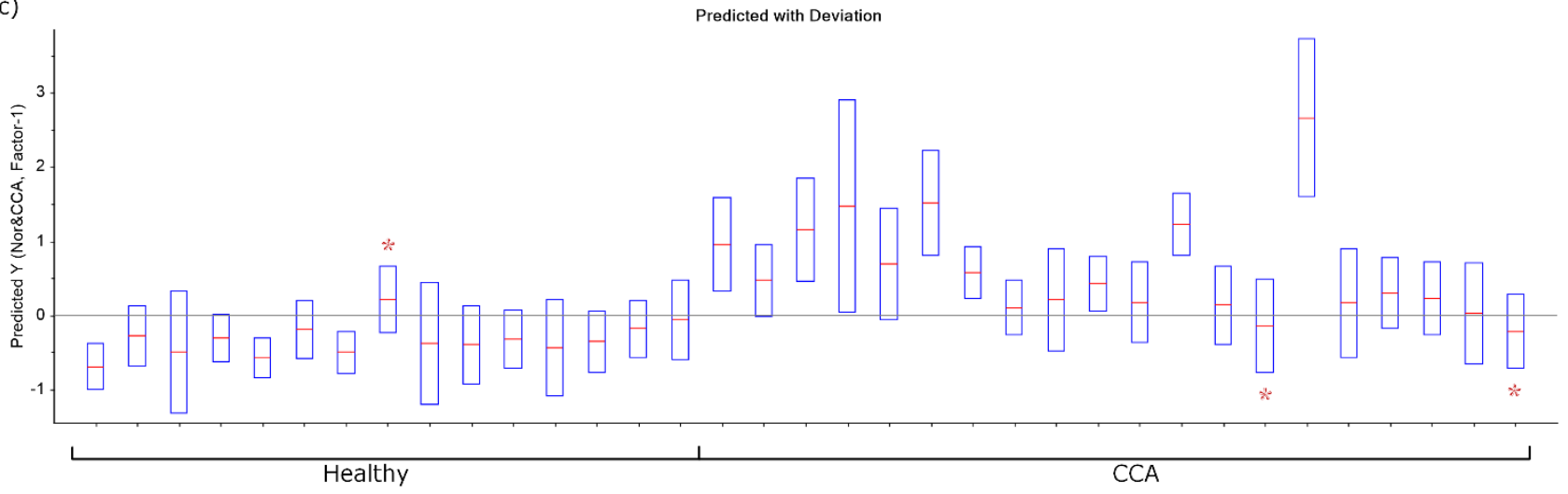

Figure 3. PLS-DA results from ATR-FTIR sera spectra, healthy (green) and CCA (red) display in (a) scores plots, (b) regression coefficients and (c) predictive result at $1400-1000 \mathrm{~cm}^{-1}$. The stars $\left(^{*}\right)$ indicate the false prediction samples in the model which give 1 false positive and 2 false negative predictions. 
Table 2. Evaluation of CCA predictive models in different spectral regions.

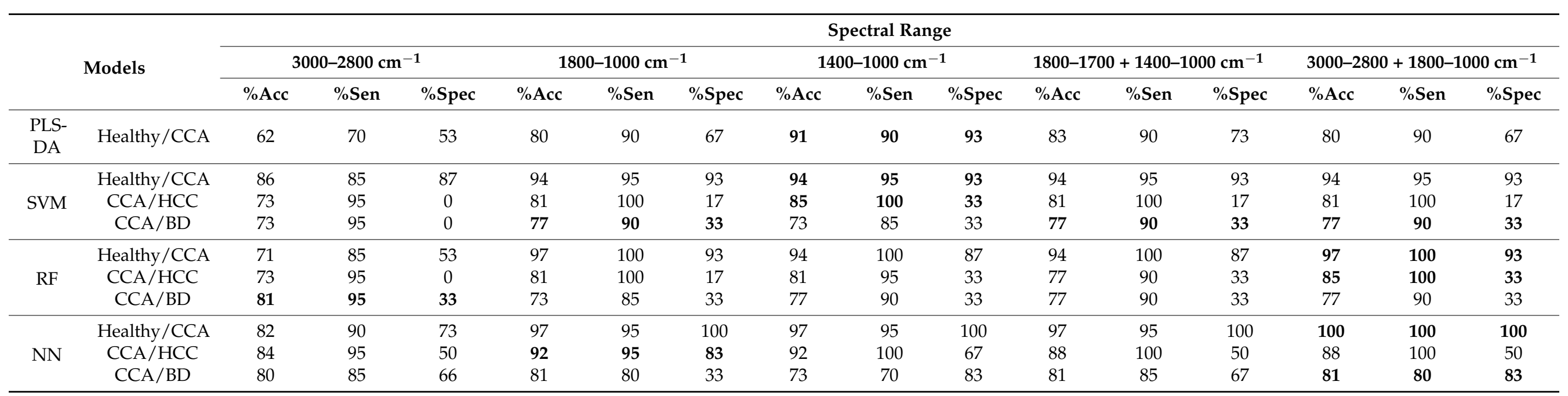

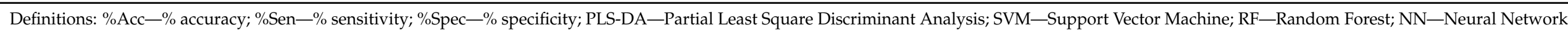

Bold words indicate the best predictive values in each model. 
According to the predictive model, the positive values were predicted as CCA, while the negative values were predicted as healthy. The modelling performed in five spectral regions, ranging from 62 to $91 \%$ accuracy, 70 to $90 \%$ sensitivity and 53 to $93 \%$ specificity. The results showed that the $1400-1000 \mathrm{~cm}^{-1}$ spectral region (Figure 3c) provided the best prediction with 14 healthy and 18 CCA, giving one false positive and two false negatives, based on the minimizing of major proteins, e.g., albumin and globulin in the amide I and II region. This indicated that the PLS-DA provided a better discrimination between healthy and CCA sera compared to the unsupervised analysis (PCA).

We further attempted to differentiate between different disease patient groups, which developed similar clinical symptoms and laboratory test results and, hence, difficult for physicians to diagnose. PLS-DA was performed on CCA vs. HCC and CCA vs. BD samples in five spectral regions. Figure S4 shows the PLS scores plots of CCA vs. HCC and CCA vs. $\mathrm{BD}$, the results indicated no discrimination among each group so a more advanced machine modelling was required to achieve the differentiation among disease groups.

\subsection{Advanced Machine Modelling of CCA Serum}

A more advanced machine learning was performed using a Support Vector Machine (SVM), Random Forest (RF) and Neural Network (NN). The models were established in five spectral ranges using vector normalized 2nd derivative spectra, $2 / 3$ of the dataset was used as the calibration set and $1 / 3$ used as the validation set. Firstly, SVM was applied as a nonlinear analyzing tool for spectral data, which contained high dimensional input attributes. A radial basis function kernel was chosen for the SVM learning. The $1400-1000 \mathrm{~cm}^{-1}$ spectral model gave the best predictive values for a differentiation of CCA sera from healthy sera with a $94 \%$ accuracy, $95 \%$ sensitivity and $93 \%$ specificity, and from HCC patients with a $85 \%$ accuracy, $100 \%$ sensitivity and $33 \%$ specificity. For a differentiation of CCA from $\mathrm{BD}$, the best prediction was obtained from three spectral regions-1800-1000 cm $\mathrm{cm}^{-1}, 1800-1700+1400-1000 \mathrm{~cm}^{-1}$ and $3000-2800+1800-1000 \mathrm{~cm}^{-1}$ 一, with equal accuracy, sensitivity and specificity at $77 \%, 90 \%$ and $33 \%$ (Table 2 ). Moreover, candidate scatter plots of 5 spectral ranges were showed in Table S1. Although SVM had an improved better sensitivity to discriminate CCA from other groups, the specificity was limited.

To obtain a better specificity, other learning algorithms were applied to analyze these spectral data. The analysis using RF was performed with a bagging learner, 100 iterations and 100 batch sizes using a 10-fold cross-validation. The best predictive values for a differentiation of CCA from healthy and HCC obtained by using the combined regions, $3000-2800+1800-1000 \mathrm{~cm}^{-1}$, resulted in an equal $100 \%$ sensitivity with $93 \%$ and $33 \%$ specificity, respectively. For the CCA and BD model, the $3000-2800 \mathrm{~cm}^{-1}$ spectral region was found to be the best model for a differentiation with $95 \%$ sensitivity and $33 \%$ specificity. Thus, RF was still limited in specificity.

The NN analysis was finally performed by multilayer perceptron with one hidden layer, which varied the number of nodes from 0 to 35 nodes and one default parameter to identify the network which provided the best sensitivity, specificity and accuracy. Each model was set with the same parameters: 0.3 learning rate, 0.2 momentum and 500 epochs in a 10-fold cross-validation. Compared with the other advance model, $\mathrm{NN}$ improved the prediction outcome in CCA and the healthy model up to a 100\% accuracy, $100 \%$ sensitivity and $100 \%$ specificity at the combined spectral region at $3000-2800+1800-1000 \mathrm{~cm}^{-1}$; however, the $\mathrm{CH}$ stretching region $\left(3000-2800 \mathrm{~cm}^{-1}\right)$ alone resulted in the worst values. This combined spectral region with no hidden layer tended to be the best model to differentiate CCA from healthy sera samples (input: hidden node: output $=541: 0: 2)$ (Table S2).

For the CCA and HCC models, the $100 \%$ sensitivity was obtained at the $1400-1000 \mathrm{~cm}^{-1}$ and the combined spectral regions, but with a rather low specificity. The best compromised model at $1800-1000 \mathrm{~cm}^{-1}$ (input: hidden node: output = 432: 2: 2) was suggested with a $92 \%$ accuracy, $95 \%$ sensitivity and $83 \%$ specificity. In the CCA and BD model, the spectral 
regions $3000-2800+1800-1000 \mathrm{~cm}^{-1}$ gave the highest accuracy, sensitivity and specificity with $81 \%, 80 \%$ and $83 \%$, respectively (input:hidden node:output $=541: 14: 2$ ).

\section{Discussion}

In our previous study [18], we reported the discrimination of O. viverrini + NDMA infected from uninfected hamster sera using PCA in the fingerprint spectral region $\left(1800-900 \mathrm{~cm}^{-1}\right)$. The important spectral signatures included: (i) a band at $1745 \mathrm{~cm}^{-1}$ assigned to the lipid ester carbonyl $\mathrm{C}=\mathrm{O}$, (ii) bands at $1380-1200 \mathrm{~cm}^{-1}$ and $1034 \mathrm{~cm}^{-1}$ from collagen, (iii) a band at $1071 \mathrm{~cm}^{-1}$ from nucleic acid phosphodiester groups and iv) a band at $1153 \mathrm{~cm}^{-1}$ from polysaccharide molecules (Table 3). These bands were also observed in the current study and compared with the animal study in Table 3.

The band at $\sim 1074 \mathrm{~cm}^{-1}$ observed in serum was tentatively assigned to circulating tumor DNA (ctDNA) fragments that were characteristic of cancer and were released into the blood stream [11,24] or, alternatively, from phosphorylated proteins, which were also found in the serum [25]. The observed changes in the carbohydrate region $1300-1000 \mathrm{~cm}^{-1}$ could be explained by two phenomena: the changes in the sugar backbone of nucleic acids and an elevation in carbohydrates [26] and the byproduct of glucose consumption or glycogen degradation related to cancer metabolism and cancer progression [27-29]. Furthermore, collagen released from the tumor microenvironment could be indicative of cancer progression and, thus, be a prognostic indicator for cancer [30,31]. Prakobwong et al. reported a significantly increase in type I collagen, hydroxyproline (HYP), metrixmetalloproteinase-7 (MMP-7) and the tissue inhibitor of MMP-2 (TIMP-2) levels in CCA plasma compared to healthy and benign biliary disease [32]. The specific protein spectral signatures in sera could be derived from the discharge of some protein from the tumor microenvironment [33]. Additionally, the tumor markers, which were found in the circulating system, could be CA19-9, $\alpha 1 \beta$-glycoprotein, afamin, MMP-7, HYP, collagen I and $\gamma$-Glutamyltransferase, etc. [8].

Table 3. FTIR spectral markers important in the discrimination of CCA from healthy human serum and hamster serum using PCA and PLS-DA.

\begin{tabular}{|c|c|c|c|c|c|c|}
\hline \multirow{3}{*}{ Biomolecule } & \multirow{3}{*}{$\begin{array}{l}\text { Molecular } \\
\text { Vibration }\end{array}$} & \multicolumn{4}{|c|}{ Wavenumber $\left(\mathrm{cm}^{-1}\right)$} & \multirow{3}{*}{ References } \\
\hline & & \multicolumn{2}{|c|}{ PCA } & \multicolumn{2}{|c|}{ PLS-DA } & \\
\hline & & Human Serum & $\begin{array}{c}\text { Hamster } \\
\text { Serum }\end{array}$ & Human Serum & $\begin{array}{c}\text { Hamster } \\
\text { Serum }\end{array}$ & \\
\hline Lipid & $\mathrm{C}=\mathrm{O}$ & 1747 & 1745 & 1743 & 1736 & {$[34,35]$} \\
\hline \multirow{2}{*}{ Collagen } & \multirow{2}{*}{$\begin{array}{l}\text { Amide III and } \\
\mathrm{CH}_{2} \text { wagging } \\
\mathrm{CH}_{2} \text { vibration }\end{array}$} & & $1380-1200$ & $1380-1200$ & $1380-1200$ & [36-40] \\
\hline & & $\begin{array}{l}1339 \\
1035\end{array}$ & $\begin{array}{c}\sim 1337 \\
1030\end{array}$ & $\begin{array}{l}1337 \\
1034\end{array}$ & $\begin{array}{c}\sim 1337 \\
1030\end{array}$ & [41] \\
\hline $\begin{array}{l}\text { Nucleic acid or } \\
\text { Protein }\end{array}$ & $v_{s} P_{2}^{-}$ & 1073 & 1077 & 1074 & 1076 & {$[23,25,34,42]$} \\
\hline Polysaccharide & C-O stretching & 1152 & 1156 & 1154 & 1153 & {$[22,23]$} \\
\hline
\end{tabular}

The infrared spectral signatures corresponding to CCA presented in this study were obtained from the comparison of CCA and healthy sera. However, these spectral peaks can be observed in various diseases, e.g., the study of cirrhosis and HCC compared to healthy sera by Thumanu et al. [43]. The abnormalities in liver can be observed in sera spectra via an alteration of a protein secondary structure and higher lipid accumulation. They reported a decrease in $\alpha$-helix at $1648 \mathrm{~cm}^{-1}$ and an increase in the $\beta$-sheet secondary structure of protein at $1639 \mathrm{~cm}^{-1}$ in HCC and cirrhosis. Additionally, the peak at $\sim 1745 \mathrm{~cm}^{-1}$ was found in cirrhosis sera which confirmed a state of a high lipid level. Sitnikova et al. [44] also reported the significance differences between healthy and breast cancer sera in the spectral range $1306-1250 \mathrm{~cm}^{-1}$ corresponding to the vibrations of several functional groups 
of DNA and RNA. Our results also agreed with a recent study on sera from brain cancer patients using ATR spectroscopy, with changes in the $1540-1490 \mathrm{~cm}^{-1}$ and $1036 \mathrm{~cm}^{-1}$ regions being observed [45]. Due to the similar biochemical components associated with CCA, HCC and BD sera being closely related with each other [46], the infrared spectra also showed similar profiles and, therefore, the discrimination specificity was decreased among three groups. To enable a more robust classifier, sophisticated mathematical and chemometric methods were necessary to identify discriminating zones reflecting a different molecular composition of the spectra [47]. Consequently, more advance machine modelling was required to achieve a higher degree of differentiation.

According to the diagnostic limitation in CCA, the exact sensitivity and specificity of each diagnostic technique could not be easily determined. Thus, the integrated techniques are often used to provide a better diagnostic efficacy [48-51]. The tumor marker, CA 19-9, showed a 50-100 \% sensitivity and 50-98\% specificity in PSC patients, whereas a combination with imaging techniques showed a $78 \%$ sensitivity and $67 \%$ specificity $[52,53]$. The elevation of CEA levels, which is an indicator of glycoprotein produced by the GI tract, reported a 33-84\% sensitivity and 33-100\% specificity, while the combination of CA 19-9 and CEA levels provided a 86\% accuracy in a PSC patient [53]. Several studies have reported several efficient biomarkers for CCA diagnosis. However, the monitoring of these markers requires special equipment along with complicate analysis procedures $[8,54]$. Meanwhile, our candidate CCA spectral modelling using PLS-DA, SVM, RF and NN resulted in a 90\% sensitivity and 93\% specificity, 90-100\% sensitivity and 33-93\% specificity, 95-100\% sensitivity and 33-93\% specificity and 80-100\% sensitivity and 83-100\% specificity, respectively.

Several studies on the application of FTIR spectroscopy and chemometric analysis for cancer diagnosis using sera have been reported. For example, an ATR-FTIR study on brain cancer sera using SVM provided a high sensitivity and specificity with $93.3 \%$ and $92.8 \%$, respectively [17]. Another study evaluated the diagnostic model of brain cancer using Random Forest, PLS-DA and SVM, which provided sensitivities and specificities of 93.8\% and $80.1 \%, 95.9 \%$ and $81.7 \%$ and $92.1 \%$ and $88.7 \%$, respectively [45]. A study of breast cancer performed using PCA-LDA reported a 92\% sensitivity, 85\% specificity and $90 \%$ accuracy, while a cluster analysis gave a $96 \%$ sensitivity, $93 \%$ specificity in the $1250-685 \mathrm{~cm}^{-1}$ region [21]. Another breast cancer study reported a Neural Network modelling efficacy with a 92-95\% sensitivity and 95-100\% specificity [15]. The differentiation accuracy, test sensitivity or specificity did not only depend on the learning algorithm, but also the input attributes, complexity of data and sample size. Hitherto, there is no consensus on the best analysis method.

This study demonstrated the advantage of ATR-FTIR coupled with a multivariate analysis or advance machine modelling to differentiate the CCA and other similar conditions and, thus, showed a promising additional tool for cancer identification. The differentiation between disease (CCA) and healthy sera was more effective than among similar disease conditions (HCC and BD). CCA-specific analytes could be found in serum, which is the most complex biofluid carrying over 20,000 different proteins [11]. The FTIR spectroscopic technique was suitable for the multi-molecular biochemical analysis, where a single spectrum can provide biomolecular information on multiple analytes [12]. However, differentiation among diseases can reduce the \% specificity of the model and the judicious selection of spectral regions was required to improve the specificity.

\section{Conclusions}

The study herein demonstrated the potential of ATR-FTIR spectroscopy as a clinical tool. A spectral shift from $1289 \mathrm{~cm}^{-1}$ in the healthy group to $1284 \mathrm{~cm}^{-1}$ in CCA, HCC and $\mathrm{BD}$ was observed in 2nd derivative spectra, which indicated changes of collagen molecules in serum. Subsequently, spectral bands of collagen were confirmed at 1339 and $1035 \mathrm{~cm}^{-1}$ from both PCA and PLS-DA analyses. Moreover, $\sim 1073 \mathrm{~cm}^{-1}$ of phosphate groups, $\sim 1747 \mathrm{~cm}^{-1}$ of lipid ester carbonyl and $\sim 1152 \mathrm{~cm}^{-1}$ of polysaccharides were also 
observed in loading plots of CCA sera in both human and hamster. A spectral analysis using a multivariate analysis and machine learning tools revealed the differentiation efficacy of CCA from other underlying diseased and healthy sera. The NN provided the best sensitivity and specificity of CCA with $80-100 \%$ and $83-100 \%$ in the selected spectral model. The portable, simple procedure, rapid and robust features of ATR-FTIR make it an ideal screening technique for a serum analysis. To test the true efficacy of the approach, a large-scale clinical trial is required to assess the true specificity against other cancers.

Supplementary Materials: The following are available online at https:/ / www.mdpi.com/article/10 .3390 / cancers13205109/s1, Figure S1: PCA scores plots of healthy and CCA sera using CH stretching region, fingerprint spectral region and the combined spectral region $\left(3000-2800+1800-1000 \mathrm{~cm}^{-1}\right)$, Figure S2: PCA scores plots of CCA vs. HCC and BD sera using $\mathrm{CH}$ stretching region, fingerprint spectral region and $1400-1000 \mathrm{~cm}^{-1}$, Figure S3: PLS-DA scatter plots of healthy vs. CCA sera in fingerprint spectral region and corresponding regression coefficients for the fingerprint spectral region and the scores plots at $\mathrm{CH}$ stretching region and combine region 1800-1700 + 1400-1000 $\mathrm{cm}^{-1}, 3000-2800+1800-1000 \mathrm{~cm}^{-1}$ spectral windows, Figure S4: PLS-DA scores plots of CCA vs. $\mathrm{HCC}$ and BD sera using $\mathrm{CH}$ stretching region, fingerprint spectral region and $1400-1000 \mathrm{~cm}^{-1}$, Table S1: indicated Support Vector Machine candidate scatter plots of CCA versus healthy, HCC and $\mathrm{BD}$ in the 5 spectral regions, Table S2: number of nodes in 1 defined as the hidden layer which gave the best \% accuracy, \% sensitivity and \% specificity in each spectral range.

Author Contributions: Conceptualization, P.C., B.R.W. and P.J.; methodology, P.C. and M.W.; validation, P.T., N.J. and K.K.; formal analysis, P.C. and K.K.; investigation, P.C. and M.W.; resources, P.J.; writing-original draft preparation, P.C.; writing-review and editing, B.R.W., P.J. and N.J.; visualization, P.C.; supervision, B.R.W., K.K, N.J., M.W., P.T. and P.J.; project administration, P.J.; funding acquisition, P.J. All authors have read and agreed to the published version of the manuscript.

Funding: This research was funded by the Royal Golden Jubilee Ph.D. Program in conjunction with the Synchrotron Light Research Institute (grant no. PHD/0214/2557) to P. Jearanaikoon and P. Chatchawal). This research was also supported by the NSRF under the Basic Research Fund of Khon Kaen University under Cholangiocarcinoma Research Institute (CARI-BRF64-25).

Institutional Review Board Statement: Left-over human samples were approved for use by the ethical committees, Center for Ethics in Human Research, Khon Kaen University (HE601117).

Informed Consent Statement: Patient consent was waived due to obtained samples indicated as left-over specimens from routine investigation.

Data Availability Statement: The data presented in this study are available in this article (and Supplementary Materials).

Acknowledgments: This work was supported by the Center for Research and Development of Medical Diagnostic Laboratories (CMDL) and Center for Innovation and Standard for Medical technology and Physical Therapy (CISMaP), Faculty of Associated Medical Sciences, Khon Kaen University. Human sera supported by the Cholangiocarcinoma Research Institute (CARI), Faculty of Medicine and Community Medical Laboratory, Faculty of Associated Medical Sciences, Khon Kaen University.

Conflicts of Interest: The authors declare no conflict of interest.

\section{References}

1. Banales, J.M.; Cardinale, V.; Carpino, G.; Marzioni, M.; Andersen, J.B.; Invernizzi, P.; Lind, G.E.; Folseraas, T.; Forbes, S.J.; Fouassier, L.; et al. Expert consensus document: Cholangiocarcinoma: Current knowledge and future perspectives consensus statement from the European Network for the Study of Cholangiocarcinoma (ENS-CCA). Nat. Rev. Gastroenterol. Hepatol. 2016, 13, 261-280. [CrossRef]

2. Alsaleh, M.; Leftley, Z.; Barbera, T.A.; Sithithaworn, P.; Khuntikeo, N.; Loilome, W.; Yongvanit, P.; Cox, I.J.; Chamodol, N.; Syms, R.R.A.; et al. Cholangiocarcinoma: A guide for the nonspecialist. Int. J. Gen. Med. 2019, 12, 13-23. [CrossRef] [PubMed]

3. Kirstein, M.M.; Vogel, A. Epidemiology and Risk Factors of Cholangiocarcinoma. Visc. Med. 2016, 32, 395-400. [CrossRef] [PubMed]

4. Khan, S.A.; Tavolari, S.; Brandi, G. Cholangiocarcinoma: Epidemiology and risk factors. Liver Int. 2019, 39, 19-31. [CrossRef] 
5. Sripa, B.; Kaewkes, S.; Sithithaworn, P.; Mairiang, E.; Laha, T.; Smout, M.; Pairojkul, C.; Bhudhisawasdi, V.; Tesana, S.; Thinkamrop, B.; et al. Liver fluke induces cholangiocarcinoma. PLoS Med. 2007, 4, 1148-1155. [CrossRef] [PubMed]

6. Hennedige, T.P.; Neo, W.T.; Venkatesh, S.K. Imaging of malignancies of the biliary tract- an update. Cancer Imaging 2014, 14, 1-21. [CrossRef]

7. Zabron, A.; Edwards, R.J.; Khan, S.A. The challenge of cholangiocarcinoma: Dissecting the molecular mechanisms of an insidious cancer. DMM Dis. Model. Mech. 2013, 6, 281-292. [CrossRef]

8. Zeng, X.; Tao, H. Diagnostic and prognostic serum marker of cholangiocarcinoma (Review). Oncol. Lett. 2015, 9, 3-8. [CrossRef]

9. Kimawaha, P.; Jusakul, A.; Junsawang, P.; Thanan, R.; Titapun, A.; Khuntikeo, N.; Techasen, A. Establishment of a potential serum biomarker panel for the diagnosis and prognosis of cholangiocarcinoma using decision tree algorithms. Diagnostics 2021, 11, 589. [CrossRef]

10. Roy, S.; Perez-Guaita, D.; Bowden, S.; Heraud, P.; Wood, B.R. Spectroscopy goes viral: Diagnosis of hepatitis B and C virus infection from human sera using ATR-FTIR spectroscopy. Clin. Spectrosc. 2019, 1, 100001. [CrossRef]

11. Sala, A.; Anderson, D.J.; Brennan, P.M.; Butler, H.J.; Cameron, J.M.; Jenkinson, M.D.; Rinaldi, C.; Theakstone, A.G.; Baker, M.J Biofluid diagnostics by FTIR spectroscopy: A platform technology for cancer detection. Cancer Lett. 2020, 477, 122-130. [CrossRef]

12. $\mathrm{Su}, \mathrm{K}$. Y.; Lee, W.L. Fourier transform infrared spectroscopy as a cancer screening and diagnostic tool: A review and prospects. Cancers 2020, 12, 115. [CrossRef]

13. Berisha, S.; Lotfollahi, M.; Jahanipour, J.; Gurcan, I.; Walsh, M.; Bhargava, R.; van Nguyen, H.; Mayerich, D. Deep learning for FTIR histology: Leveraging spatial and spectral features with convolutional neural networks. Analyst 2019, 144, 1642-1653. [CrossRef] [PubMed]

14. Lasch, P.; Stämmler, M.; Zhang, M.; Baranska, M.; Bosch, A.; Majzner, K. FT-IR Hyperspectral Imaging and Artificial Neural Network Analysis for Identification of Pathogenic Bacteria. Anal. Chem. 2018, 90, 8896-8904. [CrossRef] [PubMed]

15. Backhaus, J.; Mueller, R.; Formanski, N.; Szlama, N.; Meerpohl, H.G.; Eidt, M.; Bugert, P. Diagnosis of breast cancer with infrared spectroscopy from serum samples. Vib. Spectrosc. 2010, 52, 173-177. [CrossRef]

16. Toraman, S.; Girgin, M.; Üstündağ, B.; Türkoğlu, İ. Classification of the likelihood of colon cancer with machine learning techniques using FTIR signals obtained from plasma. Turkish J. Electr. Eng. Comput. Sci. 2019, 27, 1765-1779. [CrossRef]

17. Butler, H.J.; Brennan, P.M.; Cameron, J.M.; Finlayson, D.; Hegarty, M.G.; Jenkinson, M.D.; Palmer, D.S.; Smith, B.R.; Baker, M.J. Development of high-throughput ATR-FTIR technology for rapid triage of brain cancer. Nat. Commun. 2019, 10, 1-9. [CrossRef] [PubMed]

18. Chatchawal, P.; Wongwattanakul, M.; Tippayawat, P.; Jearanaikoon, N.; Jumniansong, A.; Boonmars, T.; Jearanaikoon, P.; Wood, B.R. Monitoring the Progression of Liver Fluke-Induced Cholangiocarcinoma in a Hamster Model Using Synchrotron FTIR Microspectroscopy and Focal Plane Array Infrared Imaging. Anal. Chem. 2020, 92, 15361-15369. [CrossRef]

19. Hackshaw, K.V.; Miller, J.S.; Aykas, D.P.; Rodriguez-Saona, L. Vibrational spectroscopy for identification of metabolites in biologic samples. Molecules 2020, 25, 4725. [CrossRef] [PubMed]

20. Barth, A. Infrared spectroscopy of proteins. Biochim. Biophys. Acta Bioenerg. 2007, 1767, 1073-1101. [CrossRef]

21. Elmi, F.; Movaghar, A.F.; Elmi, M.M.; Alinezhad, H.; Nikbakhsh, N. Application of FT-IR spectroscopy on breast cancer serum analysis. Spectrochim. Acta Part A Mol. Biomol. Spectrosc. 2017, 187, 87-91. [CrossRef]

22. Krafft, C.; Shapoval, L.; Sobottka, S.B.; Schackert, G.; Salzer, R. Identification of primary tumors of brain metastases by infrared spectroscopic imaging and linear discriminant analysis. Technol. Cancer Res. Treat. 2006, 5, 291-298. [CrossRef]

23. Movasaghi, Z.; Rehman, S.; Rehman, I.U. Fourier transform infrared (FTIR) spectroscopy of biological tissues. Appl. Spectrosc. Rev. 2008, 43, 134-179. [CrossRef]

24. Paraskevaidi, M.; Morais, C.L.M.; Raglan, O.; Lima, K.M.G.; Paraskevaidis, E.; Martin-Hirsch, P.L.; Kyrgiou, M.; Martin, F.L. Aluminium foil as an alternative substrate for the spectroscopic interrogation of endometrial cancer. J. Biophotonics $2018,11$. [CrossRef] [PubMed]

25. Zawadzka, A.M.; Schilling, B.; Cusack, M.P.; Sahu, A.K.; Drake, P.; Fisher, S.J.; Benz, C.C.; Gibson, B.W. Phosphoprotein Secretome of Tumor Cells as a Source of Candidates for Breast Cancer Biomarkers in Plasma. Mol. Cell. Proteom. 2014, 13, 1034. [CrossRef]

26. Bergquist, J.R.; Ivanics, T.; Storlie, C.B.; Groeschl, R.T.; Tee, M.C.; Habermann, E.B.; Smoot, R.L.; Kendrick, M.L.; Farnell, M.B.; Roberts, L.R.; et al. Implications of CA19-9 elevation for survival, staging, and treatment sequencing in intrahepatic cholangiocarcinoma: A national cohort analysis. J. Surg. Oncol. 2016, 114, 475-482. [CrossRef] [PubMed]

27. Duan, W.; Shen, X.; Lei, J.; Xu, Q.; Yu, Y.; Li, R.; Wu, E.; Ma, Q. Hyperglycemia, a neglected factor during cancer progression. Biomed. Res. Int. 2014. [CrossRef]

28. Zois, C.E.; Harris, A.L. Glycogen metabolism has a key role in the cancer microenvironment and provides new targets for cancer therapy. J. Mol. Med. 2016, 94, 137-154. [CrossRef] [PubMed]

29. Wei, Y.; Xu, H.; Dai, J.; Peng, J.; Wang, W.; Xia, L.; Zhou, F. Prognostic Significance of Serum Lactic Acid, Lactate Dehydrogenase, and Albumin Levels in Patients with Metastatic Colorectal Cancer. Biomed. Res. Int. 2018, 2018. [CrossRef]

30. Willumsen, N.; Ali, S.M.; Leitzel, K.; Drabick, J.J.; Yee, N.; Polimera, H.V.; Nagabhairu, V.; Krecko, L.; Ali, A.; Maddukuri, A.; et al. Collagen fragments quantified in serum as measures of desmoplasia associate with survival outcome in patients with advanced pancreatic cancer. Sci. Rep. 2019, 9, 1-8. [CrossRef] [PubMed]

31. Brassart-Pasco, S.; Brézillon, S.; Brassart, B.; Ramont, L.; Oudart, J.B.; Monboisse, J.C. Tumor Microenvironment: Extracellular Matrix Alterations Influence Tumor Progression. Front. Oncol. 2020, 10, 1-13. [CrossRef] 
32. Prakobwong, S.; Charoensuk, L.; Hiraku, Y.; Pinlaor, P.; Pairojkul, C. Plasma hydroxyproline, MMP-7 and collagen I as novel predictive risk markers of hepatobiliary disease-associated cholangiocarcinoma. Int. J. Cancer 2012, 131, 416-424. [CrossRef]

33. De Bruyne, S.; Speeckaert, M.M.; Delanghe, J.R. Applications of mid-infrared spectroscopy in the clinical laboratory setting. Crit. Rev. Clin. Lab. Sci. 2018, 55, 1-20. [CrossRef]

34. Holman, H.Y.N.; Martin, M.C.; Blakely, E.A.; Bjornstad, K.; Mckinney, W.R. IR spectroscopic characteristics of cell cycle and cell death probed by synchrotron radiation based Fourier transform IR spectromicroscopy. Biopolym. Biospectroscopy Sect. 2000, 57, 329-335. [CrossRef]

35. Notarstefano, V.; Sabbatini, S.; Conti, C.; Pisani, M.; Astolfi, P.; Pro, C.; Rubini, C.; Vaccari, L.; Giorgini, E. Investigation of human pancreatic cancer tissues by Fourier Transform Infrared Hyperspectral Imaging. J. Biophotonics 2020, 13, 1-10. [CrossRef]

36. Stelling, A.L.; Toher, D.; Uckermann, O.; Tavkin, J.; Leipnitz, E.; Schweizer, J.; Cramm, H.; Steiner, G.; Geiger, K.D.; Kirsch, M. Infrared Spectroscopic Studies of Cells and Tissues: Triple Helix Proteins as a Potential Biomarker for Tumors. PLoS ONE 2013, 8, 1-11. [CrossRef]

37. Liu, K.Z.; Dixon, I.M.C.; Mantsch, H.H. Distribution of collagen deposition in cardiomyopathic hamster hearts determined by infrared microscopy. Cardiovasc. Pathol. 1999, 8, 41-47. [CrossRef]

38. Liu, K.; Man, A.; Shaw, R.A. Molecular determination of liver fibrosis by synchrotron infrared microspectroscopy. Biochim. Biophys. Acta Biomembr. 2006, 1758, 960-967. [CrossRef] [PubMed]

39. Barnas, E.; Skret-Magierlo, J.; Skret, A.; Kaznowska, E.; Depciuch, J.; Szmuc, K.; Łach, K.; Krawczyk-Marć, I.; Cebulski, J. Simultaneous FTIR and Raman Spectroscopy in Endometrial Atypical Hyperplasia and Cancer. Int. J. Mol. Sci. 2020, 21, 4828. [CrossRef] [PubMed]

40. Belbachir, K.; Noreen, R.; Gouspillou, G.; Petibois, C. Collagen types analysis and differentiation by FTIR spectroscopy. Anal. Bioanal. Chem. 2009, 395, 829-837. [CrossRef] [PubMed]

41. Callery, E.L.; Morais, C.L.M.; Paraskevaidi, M.; Brusic, V.; Vijayadurai, P.; Anantharachagan, A.; Martin, F.L.; Rowbottom, A.W. New approach to investigate Common Variable Immunodeficiency patients using spectrochemical analysis of blood. Sci. Rep. 2019, 9, 1-15. [CrossRef] [PubMed]

42. Al-Jorani, K.; Rüther, A.; Martin, M.; Haputhanthri, R.; Deacon, G.B.; Li, H.L.; Wood, B.R. The application of ATR-FTIR spectroscopy and the reversible DNA conformation as a sensor to test the effectiveness of platinum(II) anticancer drugs. Sensors 2018, 18, 4297. [CrossRef] [PubMed]

43. Thumanu, K.; Sangrajrang, S.; Khuhaprema, T.; Kalalak, A.; Tanthanuch, W.; Pongpiachan, S.; Heraud, P. Diagnosis of liver cancer from blood sera using FTIR microspectroscopy: A preliminary study. J. Biophotonics 2014, 7, 222-231. [CrossRef]

44. Sitnikova, V.E.; Kotkova, M.A.; Nosenko, T.N.; Kotkova, T.N.; Martynova, D.M.; Uspenskaya, M.V. Breast cancer detection by ATR-FTIR spectroscopy of blood serum and multivariate data-analysis. Talanta 2020, 214, 120857. [CrossRef] [PubMed]

45. Cameron, J.M.; Butler, H.J.; Smith, B.R.; Hegarty, M.G.; Jenkinson, M.D.; Syed, K.; Brennan, P.M.; Ashton, K.; Dawson, T.; Palmer, D.S.; et al. Developing infrared spectroscopic detection for stratifying brain tumour patients: Glioblastoma multiforme: Vs. lymphoma. Analyst 2019, 144, 6736-6750. [CrossRef]

46. Giannini, E.G.; Testa, R.; Savarino, V. Liver enzyme alteration: A guide for clinicians. Cmaj 2005, 172, 367-379. [CrossRef]

47. Zhang, X.; Thiéfin, G.; Gobinet, C.; Untereiner, V.; Taleb, I.; Bernard-Chabert, B.; Heurgué, A.; Truntzer, C.; Ducoroy, P.; Hillon, P.; et al. Profiling serologic biomarkers in cirrhotic patients via high-throughput Fourier transform infrared spectroscopy: Toward a new diagnostic tool of hepatocellular carcinoma. Transl. Res. 2013, 162, 279-286. [CrossRef]

48. Khan, S.A.; Davidson, B.R.; Goldin, R.; Pereira, S.P.; Rosenberg, W.M.C.; Taylor-Robinson, S.D.; Thillainayagam, A.V.; Thomas, H.C.; Thursz, M.R.; Wasan, H.; et al. Guidelines for the diagnosis and treatment of cholangiocarcinoma: Consensus document. Gut 2002, 51, 1-9. [CrossRef]

49. Slattery, J.M.; Sahani, D.V. What Is the Current State-of-the-Art Imaging for Detection and Staging of Cholangiocarcinoma? Oncologist 2006, 11, 913-922. [CrossRef] [PubMed]

50. Blechacz, B.R.A.; Gores, G.J. Cholangiocarcinoma: Advances in Pathogenesis, Diagnosis, and Treatment. Hepatology 2008, 48, 308-321. [CrossRef] [PubMed]

51. Breitenstein, S.; Apestegui, C.; Clavien, P.A. Positron emission tomography (PET) for cholangiocarcinoma. Hpb 2008, 10, 120-121. [CrossRef] [PubMed]

52. Tshering, G.; Dorji, P.W.; Chaijaroenkul, W.; Na-Bangchang, K. Biomarkers for the diagnosis of cholangiocarcinoma: A systematic review. Am. J. Trop. Med. Hyg. 2018, 98, 1788-1797. [CrossRef]

53. Beers, B.E.V.A.N. Diagnosis of cholangiocarcinoma. HPB 2008, 10, 87-93. [CrossRef]

54. Tolek, A.; Wongkham, C.; Proungvitaya, S.; Silsirivanit, A.; Roytrakul, S.; Khuntikeo, N.; Wongkham, S. Serum $\alpha 1 \beta$-glycoprotein and afamin ratio as potential diagnostic and prognostic markers in cholangiocarcinoma. Exp. Biol. Med. 2012, 237, 1142-1149. [CrossRef] [PubMed] 